\title{
Detecting Selfish Exploitation of Carrier Sensing in 802.11 Networks
}

\author{
Konstantinos Pelechrinis*, Guanhua Yan ${ }^{\ddagger}$, Stephan Eidenbenz ${ }^{\ddagger}$, Srikanth V. Krishnamurthy* \\ *University of California, Riverside ${ }^{\ddagger}$ Los Alamos National Laboratory \\ \{kpele, krish\}@cs.ucr.edu_\{ghyan,eidenben\}@lanl.gov
}

\begin{abstract}
Recently, tuning the clear channel assessment (CCA) threshold in conjunction with power control has been considered for improving the performance of Wireless LANs. However, CCA tuning can be exploited by selfish nodes in order to obtain an unfair share of the available bandwidth. In particular, by increasing the CCA threshold, a selfish client can manipulate the carrier sensing mechanism to ignore the presence of other transmissions on the medium; consequently, it increases the probability of accessing the medium and therefore obtains a higher, unfair share of the available bandwidth. In this paper, we propose a novel approach to detect this misbehavior in WLANs. A key insight that leads to our approach is that a misbehaving node that has increased its CCA is unlikely to recognize low power receptions as legitimate packets; by intelligently sending low power probe messages, an AP can detect a misbehaving node with high probability. In a nutshell, our contributions are as follows: (a) We are the first to quantify the impact of selfish CCA tuning via extensive experimentation (b) We propose a novel lightweight scheme for detecting selfish nodes that inappropriately increase their CCA thresholds; we call our scheme CMD (for Carrier sensing Misbehavior Detection) (c) We perform extensive evaluations on an indoor 802.11 WLAN testbed to demonstrate that CMD detects misbehaving users with very high accuracy (approximately $95 \%$ of the time). Furthermore, it only incurs a false positive rate of less than $5 \%^{1}$.
\end{abstract}

\section{INTRODUCTION}

It is well known that the distributed coordination function (DCF) of the IEEE 802.11 MAC protocol provides long term fairness to the users that are in the proximity of one another and share the wireless medium [1]. Recently, there have been many approaches that advocate the joint tuning of the transmission power and the clear channel assessment (CCA) threshold to improve spatial reuse and thereby, the achievable capacity in a WLAN [2][3]. Tuning the CCA threshold opens the door for a new kind of selfish or malicious behavior. By increasing the CCA threshold, a "misbehaving" user" will cause the carrier sensing at the MAC layer to ignore the transmissions of other users with which, it shares the medium. As a consequence, (a) it may initiate transmissions when other transmissions are in progress thereby increasing collisions and, (b) it will not freeze its back-off counter while other nodes are transmitting packets; as a consequence it is able to access the medium much more frequently than other users and thus, enjoy a higher unfair share of the bandwidth. Given these adverse effects, it becomes critical that such misbehaving nodes are identified. In this paper, we propose a novel approach for detecting such nodes with high accuracy.

There are two observations that drive our approach. First, a misbehaving node that increases its CCA threshold is likely to have a good "link" to the AP to begin with. If this is not the case i.e., the misbehaving node has a poor link to the AP,

\footnotetext{
${ }^{1}$ This work was done partially with support from the US Army Research Office under the Multi-University Research Initiative (MURI) grants W911NF07-1-0318 and the NSF NeTS:WN / Cyber trust grant 0721941.

${ }^{2}$ We use the terms misbehaving, cheating, greedy and selfish interchangeably. We also use the terms user, node and client interchangeably.
}

increasing the CCA can compromise the connectivity of the node; in other words, in lieu of gaining throughput, it will lose connectivity with the AP. Second, by increasing the CCA threshold towards gaining an unfair share of the throughput, the misbehaving node implicitly raises the bar with regards to the RSSI (Received Signal Strength Indicator) required for correct decoding. The receiver circuitry only tries to decode packets that are received with an RSSI that is higher than the CCA threshold. By increasing this threshold, packets should now be received with a higher RSSI value.

Based on the above observations, we design the Carrier sensing Misbehavior Detection (CMD) system. The key insight, evident from the above observations, is that a node that has increased its CCA threshold is unlikely to correctly recognize low power transmissions from the AP as legitimate packets. Thus, by sending low power probes, the AP can potentially detect such nodes with high accuracy. In order to reduce the overhead that will be incurred due to such probes, CMD first identifies the set of possible badly behaving nodes. This set consists of those nodes that are enjoying a significantly higher share of the throughput than their counterparts that are within the same cell. The probe messages are then only sent to the members of this set. Note here that, under saturated conditions where this problem is likely to be most critical, this set naturally excludes nodes that are at the periphery of the cell or nodes with poor links. Furthermore, as stated above, a node with a poor link is unlikely to be able to increase its throughput share via the malicious behavior considered.

In more detail our contributions in this paper are as follows:

- We experimentally quantify the impact of selfish CCA tuning on the overall network performance. While previous studies have considered the benign use of CCA tuning to improve network performance, this is the first study that quantifies the extent to which, fairness suffers if this functionality were to be used inappropriately.

- We design and implement CMD for detecting such misbehaving nodes. CMD consists of two sub-components: (a) The Throughput Monitoring Module (TMM), which identifies a candidate set of possible misbehaving nodes and (b) The Low power Probing Module (LPM), which transmits the low power probes to effectively detect the real misbehaving nodes from among this candidate set. The implementation of CMD does not require any modifications to the IEEE 802.11 driver or firmware and can be implemented in the user space in its entirety.

- We analytically compute system parameters for CMD such that low false positive (wrongly classifying a well-behaved node) and false negative (not recognizing a misbehaving node) probabilities are achieved. We validate our analytical results through measurements.

- We perform extensive experiments to evaluate CMD on an indoor WLAN testbed, with various configurations. Our 
experiments show that CMD detects misbehaving nodes with extremely high accuracy $(95 \%)$ with a very low false positive rate $(<5 \%)$.

Our work in perspective: Selfish behaviors that target 802.11 functionalities have been considered and addressed previously. In particular, there have been many efforts that try to overcome behaviors where greedy nodes manipulate the backoff timers with 802.11 [4][5][6][7][8][9]. While a misbehaving node can enjoy lower back-off times by manipulating the CCA threshold (lesser chances of freezing the back-off counter), we wish to point out that the two attacks are not the same (as discussed later, the previously proposed strategies cannot deal with the considered attack). In particular, unlike the other attacks, tuning the CCA threshold is protocol compliant: the 802.11 standard [10] does not specify a value for the CCA threshold. In fact, different wireless network interface cards (NICs) have slightly different CCA thresholds. Although currently, tuning the CCA threshold is a functionality that these cards implement in the firmware, there are ongoing efforts towards enabling this functionality [11]. There have already been research efforts that advocate the tuning of this threshold for performance improvements [2][3]. In addition, GNU software defined radios [12][13] are expected to fully support 802.11 soon; such coexisting platforms that allow CCA tuning could be misused to pilfer a higher share of the throughput.

Finally, note that we only consider uplink traffic since one might expect that the APs, which are usually controlled by service providers, are unlikely or do not have the incentive to cheat; stated otherwise, it is unlikely that downlink traffic will be prone to such misbehaviors. The uplink traffic of a WLAN is not a negligible percentage of the total AP traffic anymore [14]. The increasing popularity of $p 2 p$ applications (such as bit-torrent) result in a generation of a high proportion of uplink traffic in commercial hotspots.

The rest of the paper is organized as follows. In Section II we discuss relevant CSMA/CA behavior in brief and related work. In Section III we describe our testbed at UC Riverside. Our experiments to quantify the impact of the considered attack are presented in IV. In Section V we present the design and implementation of CMD; we analyze its performance in Section VI. In Section VII we discuss the results of our evaluations of CMD. Miscellaneous issues are deliberated upon in Section VIII. Our conclusions form Section IX.

\section{BACKGROUND AND RELATED WORK}

In this section we provide a brief description of relevant CSMA/CA functions and describe related work.

Relevant 802.11 functions: 802.11's access policy is based on CSMA/CA. Each user needs to sense the medium idle for a specified time prior to transmitting data [15]. Whenever the perceived power on the medium is higher than the CCA threshold, a node must defer its transmission and enter the backoff state. Upon reaching this state, a node initiates a backoff counter with a random value. For each time slot that the medium is free, the counter is decremented; for each time slot that the energy on the medium is higher than the CCA threshold, the value of a counter is left unchanged (or frozen). When the counter value is decremented to zero, the node senses the medium again. If the power on the medium is lower than the CCA threshold (medium is idle) it transmits its packet;

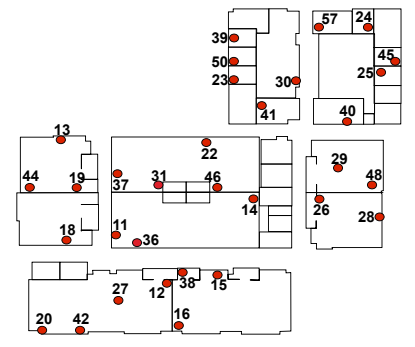

Fig. 1. The UCR wireless testbed.

otherwise, it re-enters the backoff state; the expected counter value is now doubled.

When a misbehaving node increases its CCA threshold, it can result in the following effects:

- It can now ignore those signals that it senses, but are lower than this new increased threshold. Therefore, many of the signals on the medium have now no effect on transmission opportunities of the node.

- Other nodes that use the default CCA will sense the transmissions of the selfish node and will defer their own transmissions for longer periods.

- If a transmission of the misbehaving node is not successful, it will enter the backoff state. However, since its CCA threshold is increased, it is more likely that it will not have to freeze its backoff counter; this is a consequence of the node sensing the medium to be idle even if there are ongoing transmissions.

An interesting experimental study of how carrier sensing works in practice can be found in [16].

Related studies: While there have been prior efforts on overcoming attacks that manipulate 802.11 functionalities, the attack considered in this paper has not received prior attention.

Attacks that violate the 802.11 back-off timers: Kyasanur and Vaidya [4] consider selfish behaviors where nodes deviate from the standard backoff mechanism of 802.11. They propose a mitigation scheme where the receiver explicitly assigns the backoff value to the sender. Konorski [9] proposes a misbehaviorresilient backoff mechanism. Gagalj et al [7] use game theory to develop a simple, localized and distributed protocol that guides multiple selfish nodes to a Pareto-optimal Nash equilibrium. Their work also considers attacks where nodes deviate from the backoff mechanism. Radosavac et al [5] present a framework based on Sequential Probability Ratio Test (SPRT) for detecting nodes that deviate from the backoff mechanism. Finally, Queseth [8] shows that it is hard to discourage selfishness by punishment if we cannot quickly detect these behaviors. All these studies however, are primarily related to the exploitation of the backoff mechanism, which is not the focus of our work. Note that in the considered setting, a node only increases its CCA threshold and does not violate the back-off policies; thus, these previously considered methods will not be effective.

Detecting other selfish behaviors: Raya et al [6] propose and implement DOMINO, a system for detecting various selfish behaviors in WLANs. DOMINO detects nodes that do not adhere to the standard backoff mechanism, send out data without waiting for the standard DIFS period, use an oversized NAV to retain the medium for a longer time, or intentionally corrupt frames to get more medium access. In the attack considered, misbehaving nodes increase their $\mathrm{CCA}$; none of the above 
behavioral trends are observed (as an example, the DIFS periods followed by the selfish nodes are legitimate). DOMINO cannot accurately detect an attack where nodes "do not" freeze their back off counters due to ongoing transmissions. Consequently, DOMINO cannot detect possible CCA manipulations. Note that our approach can be complementary to DOMINO.

To the best of our knowledge we are the first to examine the selfish behaviors of clients in WLANs that try to increase their throughputs by exploiting the CCA threshold functionality.

\section{EXPERIMENTAL SETUP}

In this section we provide a brief description of our testbed and the experimental methodology that is followed.

Testbed description: Our wireless testbed (Figure 1) is located on the 3rd floor of Engineering Building II at UC Riverside and consists of 32 Soekris net 4826 nodes [17]; the nodes mount a Debian Linux distribution with kernel v2.6, over NFS. Each node is equipped with two miniPCI $802.11 \mathrm{a} / \mathrm{g} \mathrm{WiFi}$ cards, an EMP-8602 $6 G$ with Atheros chipset and an Intel-2915. We use the MadWifi driver [18] for the EMP-8602 $6 G$ cards. We use a proprietary version of the ipw2200 AP and client driver/firmware of the Intel-2915 card. With this version we are able to tune the CCA threshold parameter.

Experimental methodology: For the purposes of our work we deploy the nodes of our testbed in a WLAN configuration (AP-client settings). The misbehaving clients exclusively use our Intel cards, since these cards allow us to tune CCA. The default value for the CCA threshold is $-80 \mathrm{dBm}$. All nodes use the maximum power $(18 \mathrm{dBm})$. Misbehaving nodes increase their CCA thresholds to the maximum value that guarantees association with the affiliated AP, while maintaining at least the throughput of the default settings (in isolation). We experiment with a large number of configurations; a configuration is a tuple: $<$ AP ID, Client List, Cheater $>$. We provide more details on every experiment in the following sections.

\section{The PRoblem}

The 802.11 MAC protocol, as discussed earlier, provides long term max-min fairness to nodes that share a link. Under saturated conditions all the nodes that share a link, essentially access the medium with the same probability. By increasing the CCA threshold, a node can pilfer a higher share of the medium than it is entitled to, from the other users. To reiterate, transmissions that arrive at the receiver circuitry with an RSSI lower than the CCA threshold are ignored. By increasing the threshold, a node can ignore a significant fraction of the transmissions that occupy the medium. As described before, this not only causes increased collisions but also allows the misbehaving node to reduce the fraction of the time that it spends in the back-off state.

Our objective in this section is to demonstrate the effects of this greedy behavior via an extensive set of experiments on our testbed. We experiment with various configurations (with varying locations of the APs and clients) and measure the throughput gains of the selfish clients relative to their fair share of throughputs under normal operating conditions.

Experiments with saturated traffic: We depict our first results in Figure 2. The x-axis represents the throughput gains of the selfish clients and the y-axis represents the percentage of occurrences of this throughput gain (the gains are quantized into three levels); we vary the number of clients connected to the AP. We observe that in most cases (more than $85 \%$ of the 90 scenarios in total considered) the cheating user is able to gain significantly over the well-behaved clients affiliated with the same AP - at least 5Mbps gain from its fair share.

In some scenarios though (in fewer than 5\% of the considered scenarios), the selfish client is unable to pilfer more than $2 \mathrm{Mbps}$ from the other clients. These cases arise when the selfish client is far from the AP (e.g., node 36 is the AP and node 22 is the selfish client) and as a result cannot increase its CCA to very high values; doing so would result in its disassociation from the AP. These studies suggest that a selfish node is likely to choose a location that is as close to the AP as possible ${ }^{3}$.

In Figure 3 we present the temporal variations in throughput from a representative experiment. In particular, we use node 31 as an AP and nodes 22 and 14 as clients (Figure 1). We initiate fully saturated uplink traffic from both clients using iperf for 30 seconds. During the first ten seconds, both clients enjoy the same share of the throughput; this is a direct artifact of the fairness due to CSMA/CA. In the period between the $10^{t h}$ and the $20^{\text {th }}$ seconds, node 14 misbehaves by increasing its CCA threshold from $-80 \mathrm{dBm}$ to $-50 \mathrm{dBm}$. We notice from Figure 3 that this results in a dramatic increase in the throughput of node 14. Meanwhile, node 22's throughput degrades significantly.

We observe that if the misbehavior is temporary, the effects are not long-lasting. As soon as the selfish user restores its default settings, the throughputs of the rest of the clients quickly return to the values under benign conditions. To understand this effect, recall that the selfish user follows the standard backoff mechanism with 802.11. After the settings are restored, within a short period of time, the greedy client enters the backoff state (senses energy on the medium). Then, the other users begin reducing their backoff counters; they gain access to the medium when their counter has reached the value of zero and at this point in time, fairness is restored ${ }^{4}$.

Note also that even during the period where there is selfish behavior, the well-behaved nodes still obtain some throughput; this is directly attributed to the above reason i.e., packet losses can still occur for the misbehaving node and it can still enter the back-off phase.

Behavior with TCP traffic: In the previous experiments both clients 14 and 22 had fully saturated uplink traffic, i.e., they always had packets for transmission in their MAC layer queues. Next, we consider unsaturated traffic and in particular, TCP flows. The scenario again consists of well behaved clients and a greedy user that increases its CCA threshold.

The use of TCP results in two somewhat conflicting effects from the perspective of a well-behaved user. On the one hand, since the selfish user accesses the medium more often (as discussed above), the TCP packets experience longer delays and round trip times (RTT); thus, the TCP congestion window does not increase as rapidly as one might expect under normal operations and the overall throughput suffers. On the other hand, the selfish client itself might experience loss of packets and this causes its TCP connection to reduce its congestion window. In

\footnotetext{
${ }^{3}$ Note that well behaved users may also exhibit similar behaviors in order to improve the qualities of their links to the AP; such locations can result in higher RSSI values and thus, higher transmission rates can be sustained.

${ }^{4}$ The speed with which this process occurs depends on the quality of the link between the AP and the misbehaving client. If this link is lossy, the misbehaving client is likely to experience a packet loss quickly and enter the back-off state.
} 


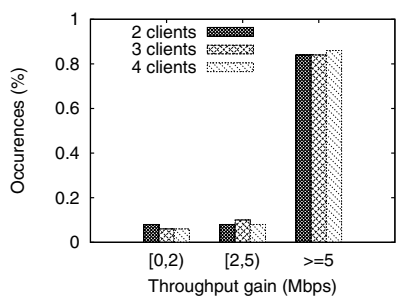

Fig. 2. Increasing CCA can be an effective greedy strategy.

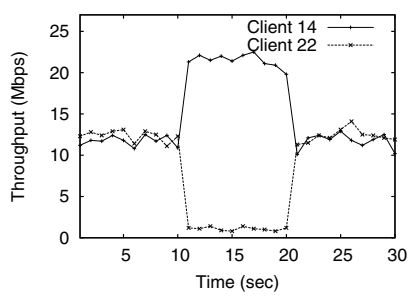

Fig. 3. Time trace for the case of UDP traffic.

other words, packet losses have an impact on the data rate with TCP and thus, the selfish user will access the medium less often than it did in the UDP scenario.

In order to quantify the impact of CCA tuning on the behaviors of transport layer protocols, we conduct a large number of experiments. We use 90 different topologies using 15 different APs with 2 clients associated with them and consider all possible combinations of the two commonly used transport layer protocols, TCP and UDP. The misbehaving node employs its greedy strategy for the entire 30 second period (abnormal operation). The results are presented in terms of the average throughputs of the well behaved and the selfish node in Figure 4; $95 \%$ confidence intervals are also shown. We also show the performance during normal operations where both clients are using the default settings. When the misbehaving client is sending UDP traffic its throughput gains are large. As one might expect, the impact is even higher when the well-behaved client is using TCP. The results show that significant gains are possible even if the link between the selfish user and the AP is lossy; this is because UDP does not reduce its sending rate upon experiencing packet losses. At the well behaved client, a lot of timeouts are triggered with TCP and the application throughput is extremely low (a few Kbps). When the misbehaving node uses TCP and the well behaved node uses UDP, the former is unable to achieve a significant gain in the throughput. This is a direct consequence of two factors (a) TCP regulates the sending rate thereby limiting the access opportunities for the selfish client and, (b) by increasing its CCA threshold, the selfish user can send more frequently, but when collisions are experienced its TCP source backs off whereas the UDP source at the wellbehaved user does not reduce its rate. When both the wellbehaved node and the selfish node use TCP, the latter benefits. Both TCP sources back-off when there are collisions; however, the selfish node is able to recover much faster since it is able to access the channel much more frequently. Figure 5 depicts the number of bytes sent per client for a representative configuration (AP-node 44, selfish client-node 13, well behaved-node 19).

To summarize, our experiments demonstrate that increasing the CCA threshold can lead to significant throughput benefits for the selfish client while hurting the other wellbehaved clients, in a majority of the cases and with different transport layer protocols.

\section{Detection SYSTEM}

In this section, we describe our scheme for detecting nodes that increase their CCA thresholds to gain an unfair throughput advantage in WLANs. We call our scheme CMD for Carrier sensing Misbehavior Detection system.

CMD is comprised of two sub-component modules: the first module, which we call TMM for Throughput Monitoring

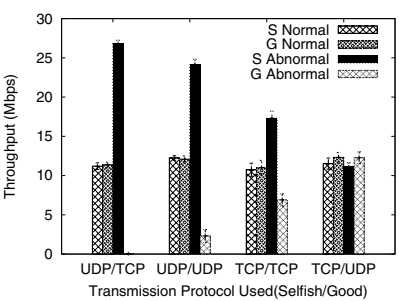

Fig. 4. Impact of different transport layer protocols (S:selfish, G:good user).

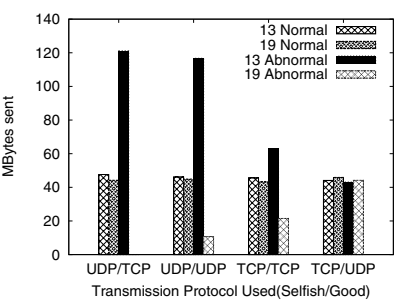

Fig. 5. Bytes sent with different transport layer protocols (AP:44, $\mathrm{S}: 13, \mathrm{G}: 19)$.
Module, aims to identify the set of potential cheating clients; note here that this set consists of those clients that are suspected of cheating but may not necessarily be real misbehavers. The second module LPM (for Low power Probing Module) tries to identify the real misbehaving clients. The key insight that motivates the design of LPM is that nodes that have increased their CCA thresholds may not be able to correctly decode low power probes.

\section{A. TMM: The Throughput Monitoring Module}

As alluded to earlier, CMD sends probes in order to achieve its goal of detecting misbehaving users. Sending probes to all the clients associated with an AP can be prohibitive in terms of overhead. The goal of TMM is to identify the nodes that could be potentially cheating by increasing their CCA thresholds. Since the IEEE 802.11 is inherently fair, a node that gets a higher share of the available bandwidth could be a potential cheater. Note that it is not necessary that a node that gets a higher share of the bandwidth is essentially a cheater since different clients might have different traffic demands; the only conclusion that one can make is that such a possibility exists.

In order to identify the nodes that have a higher share of the medium, TMM monitors the volume of uplink traffic from each and every client. A node that is able to send a much larger volume of traffic is identified as a potential miscreant.

In order to demonstrate the effectiveness of this approach in terms of including misbehaving nodes in the set output by TMM, we perform the following experiment. We set up node 31 as an AP and include 3 associated clients (nodes 14, 22 and 37 ); each client sends saturated traffic to the AP. We measure the number of packets transmitted from each client to the AP for a period of 10 seconds under two different scenarios: (a) when no client cheats and, (b) when client 37 cheats. The results are presented in Table I.

These results suggest that monitoring the traffic can be effective in identifying misbehaving nodes. However, recall that in our experiments all clients have fully saturated uplink traffic. If the clients do not have saturated traffic they may not all have the same throughput under normal operations. In particular, if one of the clients produces a higher volume of uplink traffic, it will be mistakenly classified as a cheater if we were to just use TMM to identify the misbehaving nodes. To illustrate this we perform another experiment in which the same topology as in the previous case is used. The clients are now all benign. However, they have different application data rates: client 37 sends traffic at $2 \mathrm{Mbps}$, client 22 sends at $1 \mathrm{Mbps}$ and client 14 at 24 Mbps.

Table II presents the results from this experiment. We observe that if TMM was used to classify nodes as cheaters, it would falsely conclude that client 14 is one. Thus, we need to further 


\begin{tabular}{|c|c|c|c|}
\hline Client & 14 & 22 & 37 \\
\hline Benign & 9833 & 10521 & 10461 \\
\hline Cheating & 320 & 521 & 21333 \\
\hline
\end{tabular}

TABLE I

TMM IS EFFECTIVE WITH SATURATED TRAFFIC

\begin{tabular}{|c|c|c|c|}
\hline Client & 37 & 22 & 14 \\
\hline \# Packets & 1702 & 852 & 20322 \\
\hline
\end{tabular}

TABLE II

TMM CAN BE MISLEAD WITH UNSATURATED TRAFFIC

\begin{tabular}{|c|c|c|}
\hline \# Clients & 3 & 5 \\
\hline Probing & 26.1 & 21.8 \\
\hline No Probing & 28.0 & 24.1 \\
\hline
\end{tabular}

TABLE III

OVERHEAD WITH LPM check if the nodes that are identified by TMM as potential cheaters are indeed cheaters or are legitimate recipients of higher throughputs; we do this using LPM (described later).

Implementation of TMM: We implement TMM in the user space. We develop a C application using libpcap [19]; the application is run at the AP and captures all the packets that arrive at its wireless interface. It internally maintains statistics in terms of how many packets are seen from each clients in a $Z$ second time window (we will refer to $Z$ as the monitoring window size). It then compares the number of packets from each client in order to identify the potential cheaters; if the number of packets that a client transmits, exceeds its fair share by $X$ percent (we will refer to $X$ as the deviation value), it is considered to be a possible cheater. We defer a discussion on how to choose the values of $X$ and $Z$ to Section VII.

When the potential cheaters have been identified, TMM calls LPM (described in the next section) to determine whether or not a "potential cheater" is indeed a "cheater". With this implementation we do not rely on an already available network monitoring system (for example, Ethereal and tcpdump). Instead, it computes the statistics online. In Algorithm 1, we give the high-level pseudocode of TMM.

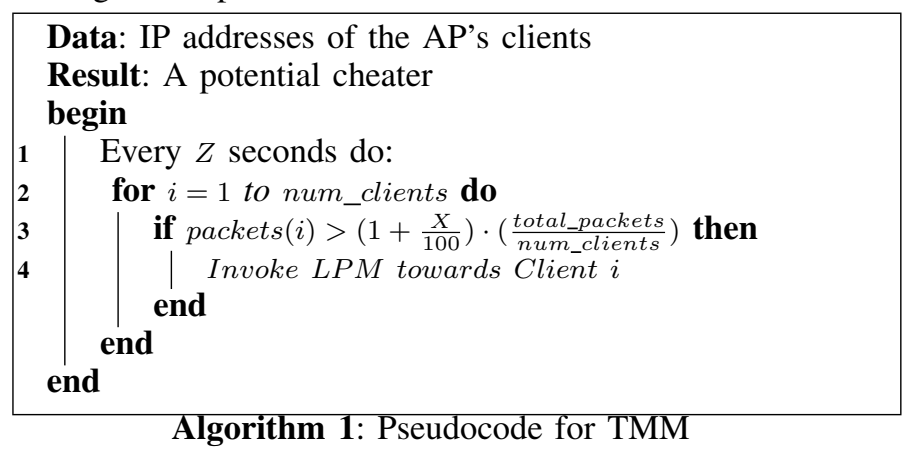

\section{B. LPM: The Low Power Probing Module}

The design of LPM is motivated by the observation that all the signals that arrive at the circuitry of a receiver with a received signal strength lower than the CCA threshold, are treated as noise; the receiver does not attempt to reconstruct packets from such signals [2]. Thus, a node that increases its CCA with the objective of increasing its throughput will not be able to correctly decode packets that are received with low powers. Thus, by having the AP probe the potential cheaters (determined by TMM) with low power packet transmissions, LPM achieves its goal of accurately identifying the real misbehaving clients.

A cheating node that increases its CCA towards obtaining a larger share of the available bandwidth, is likely to pick the maximum possible CCA without compromising on its connectivity with the $\mathrm{AP}^{5}$. The larger the CCA threshold, the higher are the number of possible ongoing transmissions that the carrier sensing logic ignores. If the CCA threshold is only increased slightly, the selfish node will not be able to achieve significant

\footnotetext{
${ }^{5}$ We assume this to be the selfish behavior for now; other possible variants are discussed in section VIII.
}

performance gains. Note here that due to this very reason, it is unlikely that nodes that are either distant from the AP (or have poor quality links) will be able to effectively launch the attack under consideration; they will not be able to increase their CCA thresholds significantly without compromising their connectivity to the AP.

Design of LPM: The new CCA threshold (chosen by a selfish node) is based on the RSSI from the AP under default operating conditions. If the AP transmits with lower powers (as compared with default settings), the RSSI value at a receiver is reduced. Going further, if this transmission power is considerably lowered, packets may arrive at the misbehaving node's antenna with an RSSI that is smaller than its increased CCA threshold. This is the key idea that drives LPM. The AP, using a reduced transmission power, sends a probe packet to each client that has been flagged as a potential misbehaving client by TMM. If a client node has increased its CCA to the extent that it exceeds the RSSI of the received probe packet, the client node cannot respond to the AP. The latter waits for a preset period of time for the client's response; if no response is received, the AP flags the client as a misbehaving node. To reduce the possibility of false alarms, LPM challenges the potential cheaters (listed by TMM) with successive ICMP_ECHO_REQUEST packets (64 bytes), sent using a reduced transmission power. The client is expected to reply to each probing packet that is received from the AP. If more than $W \%$ of the reply packets are missing from a particular client, the AP declares the client as a misbehaving client. In Section VI we discuss how we choose $W$ and the probing power such that there is a good trade-off between the false positive rate and accurate detection with our system.

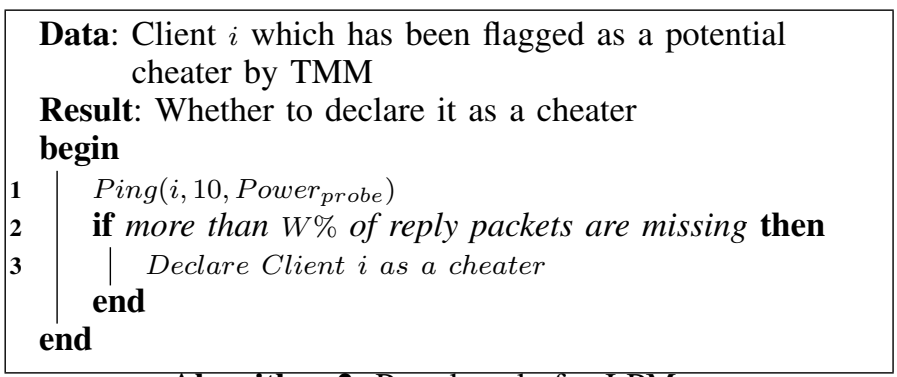

Algorithm 2: Pseudocode for LPM

TMM reduces the probing overhead due to LPM. We point out that LPM increases the overhead by sending probe packets on the medium. If the AP were to probe all the clients, then the performance degradation could be significant, especially when the number of clients is large. Table III shows the degradation in the aggregate throughput of an AP when (i) all the clients had fully saturated uplink traffic and (ii) the AP was constantly probing the clients in a round robin fashion with 10 probe packets sent each client during a probe cycle.

We observe that if there are 3 clients associated with the AP the degradation is about $7 \%$; when there are 5 clients, the degradation is about $9.5 \%$. As the number of clients increases, the degradation is higher; therefore, it is crucial to reduce the number of clients that LPM checks for real cheaters. Based on 
this, it is clear that TMM plays an important role in our system. Note also that currently, we use the 64 byte ICMP_ECHO_REQUEST messages as probes; it is possible to reduce the overhead by creating special probe messages that are of smaller size. However, this will increase the complexity of the implementation (the current implementation is described below) and may require modifications to the 802.11 driver/firmware.

Implementation details of LPM: We have implemented LPM in the user space, on top of the wireless NIC's driver. It is run at the access point. Our implementation uses a shell script that invokes the ping application [20] to probe the clients. More specifically, the script consists of a loop which parses the list of clients that are flagged as potential cheaters by TMM. We set the transmission power of the "ping" packets using the iwconfig command. Based on the results of the ping trials, LPM decides on whether a client is a cheater. This implementation is generic in that it can be run in conjunction with most commodity wireless NIC drivers.

For our Atheros cards, which use the MadWifi driver, we have also implemented our own probing utility using the Click Modular Router [21]. We use the ICMPPingSource and ICMPP ingResponder elements to implement a probe sender and a probe receiver, respectively. The Set TXPower element enables us to set the transmission power for each ICMP packet sent out by LPM. This element simply sets the Wifi TXPower Annotation flag on the packet to be sent, and we do not need to subsequently call iwconfig to set the power.

\section{An Analytical Model to Derive System PARAMETERS}

The design of LPM is based on the observation that a cheating node with an increased CCA is unlikely to respond to probe packets sent by the AP with a low transmission power. There are two cases, however, where LPM may not lead to correct diagnosis: (i) Benign clients located at the border of the AP's coverage area may not be able to respond to low power probe packets sent from the AP; these packets are likely to arrive at their circuitry with an RSSI lower than the default threshold $C C A_{d e f}$. This results in what we call false positives. (Note here that even though the links to such clients are likely to be poor, some of these clients may be getting a higher share of throughput in unsaturated traffic conditions). (ii) Misbehaving nodes could be so close to the AP that in spite of the AP using reduced transmission powers, probe packets can still reach their circuitry with an RSSI higher than their increased CCA value. In this case, the misbehaving node is not identified i.e., we have a false negative. In this section we analyze the performance of our system to determine various parametric inputs to CMD such that the false positive and false negative rates are kept low.

Propagation Model: In order to analytically determine the false positive and the false negative rates, we need to assume a propagation model. We calculate the received power $P_{r}$ at distance $r$ with transmission power $P$ to be:

$$
P_{r}=\frac{P}{r^{\alpha}} \cdot Y
$$

where $\alpha$ is the path loss exponent and $Y$ is a random variable that is log-normally distributed. The random variable $Y$ models the shadow fading effects and it has a mean value of one and a standard deviation equal to the shadow fading variation (obtained from measurements). The above model has been shown to be fairly accurate in indoor settings [22] [23].

False positives: We first compute the false-positive rate.

The probability $f(P, r)$ that a probe packet from the AP arrives at distance $r$ with an RSSI below $C C A_{d e f}$ is given by:

$$
\begin{gathered}
f(P, r)=\operatorname{Pr}\left\{\frac{P}{r^{\alpha}} \cdot Y<C C A_{\text {def }}\right\}=\operatorname{Pr}\left\{Y<\frac{C C A_{\text {def }}}{P} \cdot r^{\alpha}\right\} \\
=\frac{1}{2}+\frac{1}{2} \cdot \operatorname{erf}\left(\frac{\ln \left(\frac{C C A_{d e f} \cdot r^{\alpha}}{P}\right)-\mu}{\sigma \cdot \sqrt{2}}\right),
\end{gathered}
$$

where $\mu$ and $\sigma$ are the parameters of the log-normal distribution (computed from the mean and the measured standard deviation). We plot this probability in Figure 6. In generating this probability, the following values are used to derive the results: (i) $C C A_{d e f}=-80 \mathrm{dBm}$, (ii) the shadow fading variation is $5 \mathrm{dBm}$ (as measured from our testbed), and (iii) $\alpha=5$, which is a typical value for the path loss exponent for an indoor environment [22] [24]. The figure shows that with extreme low power operations $(1.5 \mathrm{~mW})$, the probability of violating the default CCA threshold is extremely high (false positive); with moderately low powers $(3 \mathrm{~mW})$, this same probability is almost zero upto distances of 50 meters.

Equation (2) gives the probability that a packet arrives at the client's circuitry, after traveling distance $r$, with power less than $C C A_{d e f}$. Let us assume that LPM transmits 10 probe packets and expects $n$ replies. Let $\operatorname{pr}^{p o s}(P, r, n)$ denote the probability that fewer than $n$ probe packets ${ }^{6}$ arrive at a distance $r$ with an RSSI greater than $C C A_{d e f}$. This probability is given by:

$$
p r^{p o s}(P, r, n)=\sum_{k=1}^{n}(1-f(P, r))^{k-1} \cdot f(P, r)^{10-k+1}
$$

In order to calculate the false detection rate at distance $r$ when the transmission power is $P$, we need a spatial distribution of nodes $s(r)$. As discussed in Section IV, nodes tend to stay close to the AP in reality. In order to get numerical results, a possible spatial distribution that can be used based on the previous observation is $s(r)=\frac{1}{\ln (50) \cdot r}$, for $1 \leq r \leq 50 \mathrm{~m}$ and zero otherwise ${ }^{7}$ (the constant $\frac{1}{\ln (50)}$ is chosen to assure that function $s$ is a valid probability density function). With this spatial distribution model, the false positive rate $\pi^{p o s}(P, r, n)$ at a distance $r$, when the transmission power is $P$ is given by:

$$
\pi^{\text {pos }}(P, r, n)=\left.p r^{\text {pos }}(P, r, n) \cdot s(r) \cdot \Delta r\right|_{\Delta r \rightarrow 0}
$$

We can then compute the overall false positive rate $\pi_{p}(P, n)$ when the AP is using transmission power $P$ and when LPM expects $n$ replies to its probes by integrating over the area of the cell:

$$
\pi_{p}(P, n)=\int_{0}^{\infty} p r^{p o s}(P, r, n) \cdot s(r) d r
$$

False negatives: Similar steps as above are taken in order to compute the false negative rate. However, we first need to estimate the CCA threshold, that a cheating node at distance $r$ is likely to use. The goal of the selfish client is to avoid as

\footnotetext{
${ }^{6} \mathrm{We}$ assume that the channel is reciprocal and thus, if the probe message is correctly received, the corresponding ICMP_ECHO_REPLY packet will be received with very high probability; this assumption ensures the tractability of our analysis.

${ }^{7}$ Nodes are expected to have a minimum distance -e.g. $1 \mathrm{~m}$ - from the AP which in commercial hotspots are deployed mainly on ceilings. Note that our analysis can incorporate any other spatial distribution.
} 


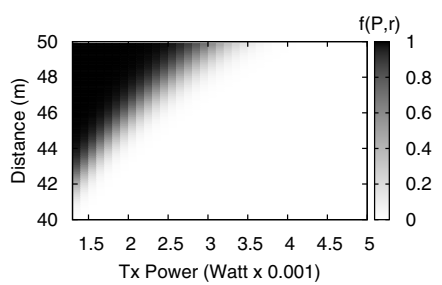

$$
\begin{aligned}
& \text { Fig. } \\
& \operatorname{Pr}\{\operatorname{signal}(P, r)
\end{aligned} \stackrel{f(P, r)}{\left.<C C A_{d e f}\right\} .}=
$$

many transmissions as possible by increasing its CCA while maintaining its connectivity with the AP (note that this is when the AP is using the default power $P_{\text {def }}$, i.e., under default operations). The CCA chosen according to this strategy can be computed by solving the following optimization problem:

$$
\begin{array}{ll}
\text { maximize } & C C A_{\text {cheat }}(r) \\
\text { subject to } & \operatorname{Pr}\left\{\frac{P_{\text {def }}}{r^{\alpha}} \cdot Y>C C A_{\text {cheat }}(r)\right\}=1 \\
& C C A_{\text {cheat }}(r) \in\{-80,-79, \ldots, 1,0\} d B m
\end{array}
$$

Solving the above optimization problem for various distances $r$, we get Figure 7 . We present in the same figure the corresponding $C C A_{\text {cheat }}(r)$ (the CCA threshold tuned as per the same strategy), measured from our testbed; for a given location of the cheater, we increase the CCA threshold to the extent possible without compromising the connectivity with the AP. The results indicate that the analytical results match reasonably well with the measurement results; the coefficient of determination $R^{2}$ [25] is calculated to be equal to 0.71 .

Having computed $C C A_{\text {cheat }}(r)$, we now proceed to calculate the false negative rate. We first calculate the probability $h(P, r)$ that a signal transmitted from the AP with power $P$ arrives at distance $r$ with a RSSI greater than $C C A_{\text {cheat }}(r)$ :

$$
\begin{gathered}
h(P, r)=\operatorname{Pr}\left\{\frac{P}{r^{\alpha}} \cdot Y>C C A_{c h}(r)\right\}=\operatorname{Pr}\left\{Y>\frac{C C A_{c h}(r)}{P} \cdot r^{\alpha}\right\} \\
=\frac{1}{2}-\frac{1}{2} \cdot \operatorname{erf}\left(\frac{\ln \left(\frac{C C A_{c h}(r) \cdot r^{\alpha}}{P}\right)-\mu}{\sigma \cdot \sqrt{2}}\right)
\end{gathered}
$$

In Figure 8 we plot $h(P, r)$ for various AP transmission powers and distances from the AP (using the same parameters as previously) and $C C A_{\text {cheat }}(r)$ computed as the solution to the optimization problem defined in (6)-(8). We observe that if the cheater is extremely close to the AP $(\approx 1 \mathrm{~m})$, there is no way of detecting it with low power probes. However, if the cheater is further than 1.5 meters, the use of a transmission power that is lower than say $3.5 \mathrm{~mW}$ can lead to an extremely high probability of detection, i.e., the probability that the signal is higher than the CCA set by the cheater is almost zero.

Given $h(P, r)$, we now calculate the probability $\operatorname{pr}^{n e g}(P, r, n)$ that no fewer than $n$ packets arrive at distance $r$ with an RSSI greater than $C C A_{\text {cheat }}(r)$ :

$$
p r^{n e g}(P, r, n)=\sum_{k=n}^{10} h(P, r)^{k} \cdot(1-h(P, r))^{10-k}
$$

Using a spatial distribution of the nodes $s(r)$, we can calculate $\pi^{n e g}(P, r, n)$, the false negative rate at distance $r$ when the transmission power of the AP is $P$ to be:

$$
\pi^{n e g}(P, r, n)=\left.p r^{n e g}(P, r, n) \cdot s(r) \cdot \Delta r\right|_{\Delta r \rightarrow 0}
$$

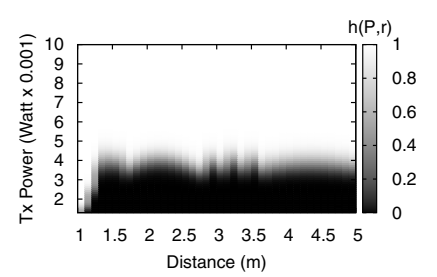

$$
\text { Fig. } \quad 8 . \quad h(P, r)=
$$$$
\operatorname{Pr}\left\{\operatorname{signal}(P, r)>C C A_{c h}\right\} \text {. }
$$

Integrating over the whole area, we get the overall false negative rate $\pi_{n}(P, n)$ when the AP transmits with power $P$ and LPM expects $n$ responses to its probes:

$$
\pi_{n}(P, n)=\int_{0}^{\infty} p r^{n e g}(P, r, n) \cdot s(r) d r
$$

Equations (5) and (12) provide the false positive and false negative rates of our system. These results also provide insights on the appropriate values for Power $_{\text {probe }}$ and $n$; these values should be chosen so as to satisfy a specific performance criterion. In short, we seek to minimize these probabilities; however, it is unlikely that they are both minimized together. Hence, we minimize the sum $\pi_{p}(P, n)+\pi_{n}(P, n)$. Solving this minimization problem yields $n=9$ and Power $_{\text {probe }}=3.3 \mathrm{~mW}$. This means that in the LPM engine we need to set $W=10 \%$ (since 10 probes were set) and Power probe $=3.3 \mathrm{~mW}$. In Figure (9) we present the ROC curve (Receiver Operating Characteristics) for the case $n=9$ and we point out the operating point which corresponds to Power $_{\text {probe }}=3.3 \mathrm{~mW}$. Each point on this curve corresponds to a different Power $_{\text {probe. Increasing }}$ Power $_{\text {probe }}$ increases false negatives; decreasing it will increase false positives. The operating point is the one that minimizes the aforementioned objective function. The corresponding false positive rate and false negative rates are: $\pi_{p}=0.0053$ and $\pi_{n}=0.054$. Note that with these settings, our detection system is able to achieve high detection accuracy.

\section{Evaluation OF CMD}

In this section, we evaluate CMD.

Evaluation of the TMM module: First, we perform experiments to evaluate how TMM performs with various combinations of its input parameters; in particular, we consider the monitoring window size $Z$ and the deviation $X \%$ from a client's fair share for it to be considered a potential cheater. Ideally, we want TMM to (i) flag all cheating nodes as potential cheaters and (ii) minimize the number of well-behaved nodes that are included in the set of potential cheaters. To evaluate the performance of TMM, we perform the following two sets of experiments.

(a) Monitoring legitimate traffic: In this set of experiments we monitor the traffic at the AP when no clients cheat and all clients have fully saturated uplink traffic. We vary both the monitoring window size $Z$ and deviation $X \%$ from each client's fair share. The false alert rate, which represents the probability that a well-behaved client is flagged as a potential cheater, is depicted in Figures 10-12; in these experiments, the numbers of clients associated with the AP are 2, 3 and 4, respectively.

From the results, we observe that when the deviation is chosen to be smaller than $20 \%$ the false alert rate can be very high, especially when the monitoring window size is small. For instance, when the deviation is set to $10 \%$ and the monitoring 


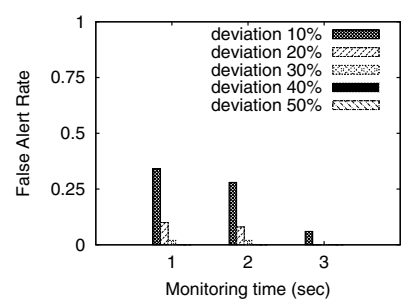

Fig. 10. TMM false alert rate when there is no selfish user (2 clients).

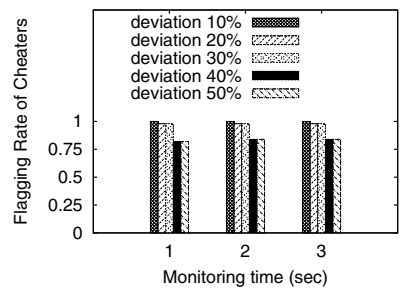

Fig. 13. TMM flagging rate of cheaters when there is a greedy client ( 2 clients).

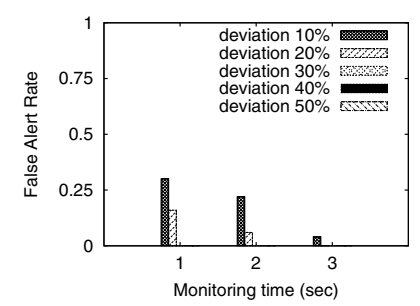

Fig. 11. TMM false alert rate when there is no selfish user (3 clients).

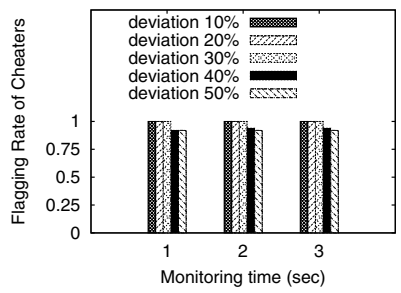

Fig. 14. TMM flagging rate of cheaters when there is a greedy client ( 3 clients).

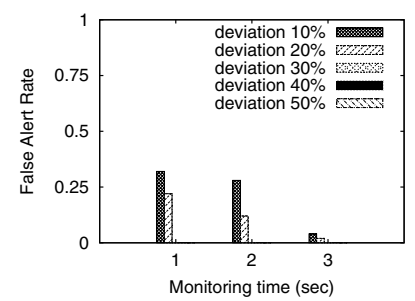

Fig. 12. TMM false alert rate when there is no selfish user (4 clients).

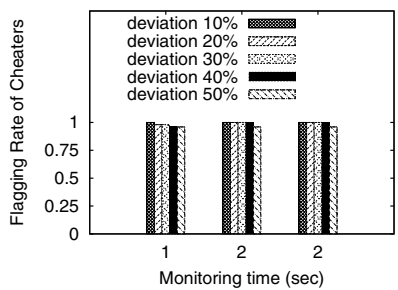

Fig. 15. TMM flagging rate of cheaters when there is a greedy client (4 clients). window size is 1 second, a well-behaved client is mistakenly flagged as a potential cheater with a likelihood of more than $30 \%$. However, if we increase the deviation, the false alert rate decreases. When the deviation is set to $30 \%$ or higher, the false alert rate is very small. The results are somewhat expected since small deviations in the expected fair share of throughput are likely; furthermore, transients are possible if the monitoring window size is not sufficiently large. Reducing the false alert rate will reduce the overhead incurred due to probing with LPM.

(b) Monitoring the cheating nodes' traffic: In this set of experiments we monitor the traffic at the AP in the presence of cheating nodes. Again, all clients have fully saturated uplink traffic. In this case, we are interested in the false negative rates of TMM; in other words, we seek to measure the probability that TMM does not include a real cheater in its output set. Figures 13-15 depict the probabilities that a cheating node is successfully identified as a potential cheater. From the results, we observe that when we use relatively small deviations (smaller than 30\%) the TMM module almost always flags the cheating node as a potential cheater. If however it uses a deviation value that is higher than $30 \%$, it misses the cheater in some cases.

The experimental results with both scenarios suggest that there is a tradeoff between the detection accuracy and the deviation value. Small deviation values help identify the cheating nodes but they may lead to high false alert rates under benign conditions; on the other hand, large deviation values help reduce the false alert rate but may miss some cheating nodes. In the current version of TMM, we set the monitoring window size to be 1 second and the deviation value to be $30 \%$. Based on the experimental results, these values achieve a good balance between the false alert rate and the false negative rate of TMM.

Evaluation of the LPM module: LPM determines whether a potential cheater reported by TMM is indeed a cheater. We perform another set of experiments to quantify its detection accuracy. We experiment with a variety of configurations that take into account both saturated and unsaturated uplink traffic. In particular we experimented with 132 configuration tuples. We utilize iperf to generate uplink traffic. The cheating node always has saturated traffic (since as discussed earlier, a misbehaving client is expected to adopt a greedy strategy in exactly these scenarios) and misbehaves shortly after the initiation of the experiment (8-10 seconds approximately). Each experiment lasts for 1 minute. We vary the transmission power of the probe packets between 3, 4, and $5 \mathrm{dBm}$. Recall that our analysis in Section VI suggests a probe power of $3.3 \mathrm{~mW}$; this corresponds to approximately $5 \mathrm{dBm}$. We compute the false positive and false negative rates with the LPM module. Note that since LPM takes the output of TMM as its input, these rates are the false detection rates for the whole system, CMD (the output of LPM is the output of CMD). The results are presented in Table IV.

\begin{tabular}{|c|c|c|}
\hline Power $_{\text {probe }}$ & False positive rate & False negative rate \\
\hline $5 \mathrm{dBm}$ & 0.015 & 0.060 \\
\hline $4 \mathrm{dBm}$ & 0.015 & 0.030 \\
\hline $3 \mathrm{dBm}$ & 0.045 & 0.015 \\
\hline
\end{tabular}

TABLE IV

DETECTION ACCURACY OF LPM

From Table IV, we note that LPM produces low false positive rates and low false negative rates in real experiments; even when the transmission power of the probe packets is varied, the maximum false positive and the maximum false negative rates are no higher than $4.5 \%$ and $6 \%$, respectively. We also observe the tradeoff between false positive rates and false negative rates as we reduce (or increase) the probing power; if we keep reducing the probing power, the false positive rate increases while the false negative rate decreases. From among the three probing powers we have used, the sum of false positive rate and the false negative rate is the smallest when Power probe is $4 \mathrm{dBm}$. This value is slightly lower than the one derived with the analysis in Section VI. The reason for this is that the assumed propagation model and its parameters (i.e., path loss exponent) or the spatial distribution of nodes $s(r)$ with the analysis, may not fit with the characteristics of our testbed with very high fidelity. Furthermore, in our analysis we focus on the performance of LPM, without considering the impact of TMM. It is hard, if not impossible, to model the interactions between the two modules accurately. This would require $s(r) \cdot \Delta r$ to include also the probability of TMM reporting a node, at distance $r$, as a potential cheater; this is difficult because it requires the knowledge of the traffic patterns of all clients (e.g., 
whether they send saturated traffic or not and their application data rates) at each location. In spite of these limitations, note that the false positive rate and false negative rate analytically derived (i.e., $\pi_{p}$ and $\pi_{n}$ in Section VI) are very close to what is observed with experimental results on the real testbed.

In our experiments LPM mistakenly declares a few wellbehaved nodes as cheaters; this happens especially when some of the clients have unsaturated uplink traffic. As discussed in Section IV, clients far away from the AP cannot gain much by applying the considered selfish strategy because they cannot increase their CCA thresholds to a significant extent. In the presence of unsaturated traffic, some well-behaved clients that are far away from the AP are wrongly flagged as potential cheaters by TMM if their application data rates are higher than that of those that are closer to the AP. Consequently with LPM, the probe packets from the AP may reach these clients with a RSSI below $C C A_{d e f}=-80 \mathrm{dBm}$. Thus, these well-behaved clients are unable to recognize these packets and send responses to the AP. However, our experiments demonstrate that such possibilities are rare given that the poor quality of the links to such clients limits the throughputs that they can achieve.

We observe that the false negative rate is about $6 \%$ when the transmission power of probe packets is $5 \mathrm{dBm}$. As we reduce this power, the false negative rate decreases significantly. For instance, when probe packets are transmitted at power $3 \mathrm{dBm}$, the false negative rate drops to about $1.5 \%$. Interestingly, if we further reduce the transmission power of probe packets to $1 \mathrm{dBm}$, all cheating nodes are successfully reported as cheaters ${ }^{8}$.

\section{Discussion}

Mitigating the effect of CCA exploitation: The goal of this work is to detect users that selfishly increase their CCA thresholds in order to get throughput gains. Mitigating the effects of such misbehaving nodes will be considered in the future; however, we deliberate on possible ways of overcoming the adverse effects of such cheaters. The simplest solution is to punish a cheating client by disassociating it completely from the AP. There are other mitigation approaches that are less harsh. As an example, the AP can choose to reduce its transmission power, which forces the cheating client to decrease its CCA threshold if it wants to communicate with the AP. Alternatively the AP may intentionally drive down the throughputs of such misbehaving clients. In particular, the AP could "not send" MAC layer ACKs to the cheating node for some of its frames. As a result, the cheating node has to back off with a larger contention window; this in turn, increases the opportunity of access to the other wellbehaved nodes. Implementation of this approach is challenging because currently most commodity NICs implement MAC layer acknowledgements in the firmware.

Response to improved cheating strategies: In Section VI we assume that a cheating node always chooses the maximum CCA threshold that guarantees its connectivity with the AP. This assumption is reasonable only if the cheating node is greedy to the maximum extent (the strategy enables the node to ignore as many transmissions as possible). If the misbehaving node knows that CMD has been deployed, it might set a CCA threshold lower than that to evade detection. Note here that a less significant increase in CCA will have a lower impact

\footnotetext{
${ }^{8}$ However we expect that such a low Power $_{\text {probe }}$ can lead to a high false positive rate.
}

on the network; thus, there is an inherent trade-off between the performance gain and the possibility of detection that the cheater has to consider. Note that it is still possible to detect misbehavior by further reducing the transmission power of the probe packets. This may lead to higher false positive rates. However, from Figure 9 we notice that even if we use the lowest transmission power considered, the false positive rate is still very low (relative to the specific spatial distribution).

\section{CONCLUSIONS}

In this paper we identify a new, powerful selfish behavior in WLANs: a misbehaving client increases its CCA to improve its chances of accessing the medium. CCA tuning has been considered previously towards providing network wide performance enhancements; this is the first study that considers the misuse of this capability. With extensive experimentation on a real testbed, we show that such selfish behaviors can cause extremely unfair allocations of the wireless medium. We develop a detection scheme that we call CMD for Carrier sensing Misbehavior Detection. We mathematically analyze its detection accuracy. We also implement CMD on an indoor wireless testbed. Through experiments we demonstrate that CMD detects such selfish clients in WLANs with extremely high accuracy and with low false positive rates.

\section{REFERENCES}

[1] M.Heusse, F.Rousseau, G.Berger-Sabbatel, and A.Duda. Performance anomaly of 802.11b. In INFOCOM, 2003.

[2] V. Mhatre, K. Papagiannaki, and F. Baccelli. Interference Mitigation through Power Control in High Density 802.11 WLANs. In IEEE INFOCOM, 2007.

[3] I. Broustis, K. Papagiannaki, S. V. Krishnamurthy, M. Faloutsos, and V. Mhatre. MDG: Measurement-Driven Guidelines for 802.11 WLAN Design. In ACM MOBICOM, 2007.

[4] P. Kyasanur and N. Vaidya. Detection and Handling of MAC layer misbehavior in wireless networks. In DSN, 2003.

[5] S. Radosavac, J. S. Baras, and I. Koutsopoulos. A framework for MAC protocol misbehavior detection in wireless networks. In WiSe, 2005.

[6] M.Raya, J-P.Hubaux, and I.Aad. DOMINO: A System to Detect Greedy Behavior in IEEE 802.11 Hotspot. In MobiSys, 2004.

[7] M. Cagalj, S. Ganeriwal, I. Aad, and J.-P. Hubaux. On selfish behavior in CSMA/CA networks. In INFOCOM, 2005.

[8] O.Queseth. The effect of selfish behavior in mobile networks using CSMA/CA. In VTC, 2005.

[9] J. Konorski. Multiple access in ad hoc wireless LANs with noncooperative stations. In NETWORKING, 2002.

[10] ANSI/IEEE 802.11-Standard. 1999 edition.

[11] Ath5k project. http://madwifi.org/wiki/About/ath5k.

[12] GNU radio trac. http://gnuradio.org/trac.

[13] USRP SDR platform. http://www.ettus.com.

[14] J.Lee, S.Choi, and H.Jung. Analysis of User Behavior and Traffic Pattern in a Large-Scale 802.11a/b Network. In WiNMee, 2005.

[15] B. O'hara and A. Petrick. IEEE 802.11 Handbook, a Designer's Companion. IEEE Press, Second Edition, ISBN 0-73-814449-5.

[16] K.Jamienson, B.Hull, A.Miu, and H.Balakrishnan. Understanding the Real-World Performance of Carrier Sense. In ACM SIGCOMM Workshops, 2005.

[17] Soekris-net4826. http://www.soekris.com/net4826.htm

[18] The MAdWiFi driver. http://madwifi.org.

[19] PCAP Unix man page. http://www.tcpdump.org/pcap3_man.html.

[20] Ping Linux Man Page. http://linux.die.net/man/8/ping.

[21] Click Modular Router. http://read.cs.ucla.edu/click/.

[22] S. Zvanovec, P. Pechac, and M. Klepal. Wireless LAN Networks Design: Site Syrvey or Propagation Models? In Radioengineering, Vol. 12, No. 4, Dec. 2003

[23] T. S. Rappaport. Wireless communications principles and practices, Prentice Hall, 2002.

[24] Path-loss. http://en.wikipedia.org/wiki/Path_loss.

[25] N.R. Draper and H. Smith. Applied Regression Analysis. WileyInterscience. ISBN 0-471-17082-8. 